\title{
Reasoning about Control Situations in Power Systems
}

\author{
Saleem, Arshad; Lind, Morten
}

Published in:

In Proceedings of 15th International Conference on Intelligent System Applications to Power Systems

Link to article, DOI:

10.1109/ISAP.2009.5352845

Publication date:

2009

Document Version

Publisher's PDF, also known as Version of record

Link back to DTU Orbit

Citation (APA):

Saleem, A., \& Lind, M. (2009). Reasoning about Control Situations in Power Systems. In In Proceedings of 15th International Conference on Intelligent System Applications to Power Systems (1 ed., pp. 1-6). IEEE. https://doi.org/10.1109/ISAP.2009.5352845

\section{General rights}

Copyright and moral rights for the publications made accessible in the public portal are retained by the authors and/or other copyright owners and it is a condition of accessing publications that users recognise and abide by the legal requirements associated with these rights.

- Users may download and print one copy of any publication from the public portal for the purpose of private study or research.

- You may not further distribute the material or use it for any profit-making activity or commercial gain

- You may freely distribute the URL identifying the publication in the public portal

If you believe that this document breaches copyright please contact us providing details, and we will remove access to the work immediately and investigate your claim 


\section{Reasoning about Control Situations in Power Systems}

\author{
Arshad Saleem \\ Department of Electrical Engineering \\ Technical University of Denmark \\ Kongens Lyngby, Denmark DK 2800 \\ Email: asa@elektro.dtu.dk
}

\author{
Morten Lind \\ Department of Electrical Engineering \\ Technical University of Denmark \\ Kongens Lyngby, Denmark DK 2800 \\ Email: mli@elektro.dtu.dk
}

\begin{abstract}
Introduction of distributed generation, deregulation and distribution of control has brought new challenges for electric power system operation, control and automation. Traditional power system models used in reasoning tasks such as intelligent control are highly dependent on the task purpose. Thus, a model for intelligent control must represent system features, so that information from measurements can be related to possible system states and to control actions. These general modeling requirements are well understood, but it is, in general, difficult to translate them into a model because of the lack of explicit principles for model construction. Available modeling concepts for intelligent control do not assist the model builder in the selection of model content i.e. in deciding what is relevant to represent for a particular reasoning task and thereby faced with a difficult interpretation problem. In this paper, we present our work on using explicit means-ends model based reasoning about complex control situations which results in maintaining consistent perspectives and selecting appropriate control action for goal driven agents.
\end{abstract}

Index Terms-Power Systems, Intelligent Control, Multiagent Systems, Means-Ends reasoning, Situation Awareness, Control Situations

\section{INTRODUCTION}

In decentralized multi-agent systems, such as de-regulated electric power systems, the world model or perspective of individual agents is based upon the goal or interest of the agent. Actions of each agent bring changes in its environment with consequences reflected in the perspective of other agents. The classic agent behaviors which are primarily based upon discrete situation-action rules may not be sufficient to cope with control situations in a dynamic environment. As an example consider the situation when a control action of the agent is failed to produce the intended result. In this case, it would be desirable that the agent could:

- Reconsider the objectives of the action and the means used

- Derive possible remedial actions and predict their consequences

- Plan and execute a (new) action

The agent may not make these decisions based on local knowledge alone and by executing behaviors based on discrete situation-action rules. It may also be necessary to consider the global situation including knowledge about the role played by the agent as member of a community of agents and the purposes and functions of the physical power system components and subsystems. Awareness about control situations can be ensured if the agent has an internal model representing the context of its actions. Ideally, the agent should not only have a library of behaviors but should also have a knowledge base representing contextual knowledge required for handling abnormal situations. Such a knowledge base representing information about the control situation in a power system can be developed using multilevel flow modeling (MFM) [10], [13], [15]. The advantage of MFM is the ability to choose level of abstraction in the model so that it matches the particular need or perspective of the agents and that relations between perspectives are logically defined. In this way it can be ensured that the perspectives of the agents are consistent and are coherent with a global perspective of the system. MFM provides concepts for semantically rich modeling of agent's context of action and mechanism to perform reasoning on this model for diagnosing and developing action plans in dynamic control situations.

\section{A. The modeling problem}

Power system models used for reasoning tasks such as intelligent control are highly dependent on the task purpose. The level of detail and abstraction of the model must comply with the needs of the task to be solved. Thus, a model for intelligent control must represent system features, so that information from power system measurements can be related to power system disturbances and possible counteractions. These general requirements to models for intelligent control are well understood, but it is in general difficult to implement the requirements into a model. The main problem is the general lack of explicit principles for model construction which take into account task requirements.

A variety of modeling concepts for intelligent control has been proposed and several types of modeling tools have been developed for representing power systems. However, these tools do not assist the modeler in solving the fundamental modeling problem which is a problem of interpretation. The model builder is not assisted in the selection of model content i.e. in deciding what is relevant to represent for a particular reasoning task and for a specific power system. The model builder is therefore faced with a difficult interpretation prob- 
lem. Within AI research this modeling challenge is referred to as the knowledge acquisition. The interpretation problem is accordingly not unique to power systems control but is a generic problem. Lind [12] discuss the modeling problem in the context of process control.

In the present paper, these interpretation problems in building models for intelligent control will be analyzed. Results of the analysis indicates that power system knowledge that can be captured in a means-end and part-whole framework.

\section{B. Multilevel Flow Modeling}

Multilevel Flow Modeling (MFM) is an approach to modeling goals and functions of complex industrial processes involving interactions between flows of mass, energy and information [8]-[10], [13], [14], [16]. MFM has been developed to support functional modeling [15] of complex dynamic processes and combines means-end analysis with whole-part decompositions to model system functions at different levels of abstraction. System functions are represented by elementary flow functions interconnected to form flow structures representing a particular goal oriented view of the system (Figure 1). Flow structures are interconnected in a multilevel representation through means-end relations, causal relations, control functions and structures. MFM is founded on fundamental concepts of action and each of the elementary flow and control functions can be seen as instances of more generic action types [14]. The views represented by the flow structures, functions, objectives and their interrelations comprise together a comprehensive model of the functional organization of the system represented as a hyper graph. It should be noted that MFM provides a formalized conceptual model of the system which supports qualitative reasoning about control situations [11], [23].

MFM has been used to represent a variety of complex dynamic processes including fossil and nuclear power generation [6], [17], [18], oil refineries [3], chemical engineering [20] and biochemical processes [1].

Application of MFM includes model based situation assessment and decision support for control room operators [19], hazop analysis [21], alarm design [24] and alarm filtering [7] and planning of control actions [2], [6]. MFM is supported by knowledge based tools for model building and reasoning [16]. The MFM concepts shown in Figure 1 will be demonstrated below with a simple modeling example.

1) An MFM example: Application of the MFM concepts shown in Figure 1 is illustrated in the following for the simple example shown in Figure 2 below. The example is a heat transfer system with a water circulation loop and associated support system for lubrication of the circulation pump. It should be noted that the example has been selected in order to serve the specific needs of the present paper. Thus we will only consider the functions involved in circulation of lube oil and the water and ignore the functions associated with the transfer of heat through the heat exchangers. By including the means-end relations between the mass flow and energy flow functions in the heat transfer system the models would have

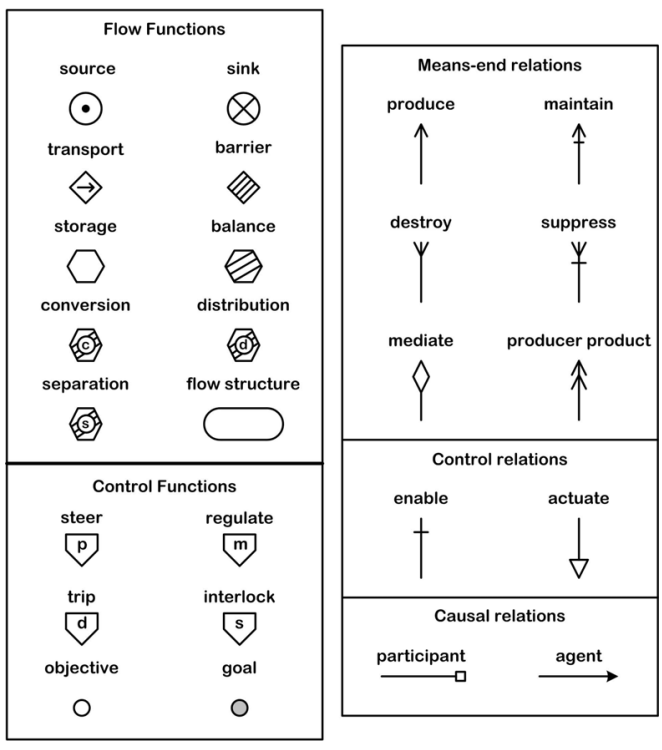

Fig. 1. MFM concepts

been more complex and representative for MFM models in general. Another aspect of MFM which of the same reason is not illustrated strongly by the example is the principal differences between physical and functional topology. The interested reader can find more complex and "interesting" examples elsewhere [1], [3], [20], [21]. An MFM model of a power system model is described below.

The water circulation loop and the lube oil system are equipped with flow measurements FM1 and FM2 and associated controllers CON1 and CON2 dealing with lube oil and water flow regulation. The purpose of the example is to demonstrate how control and process functions are integrated in the MFM models.

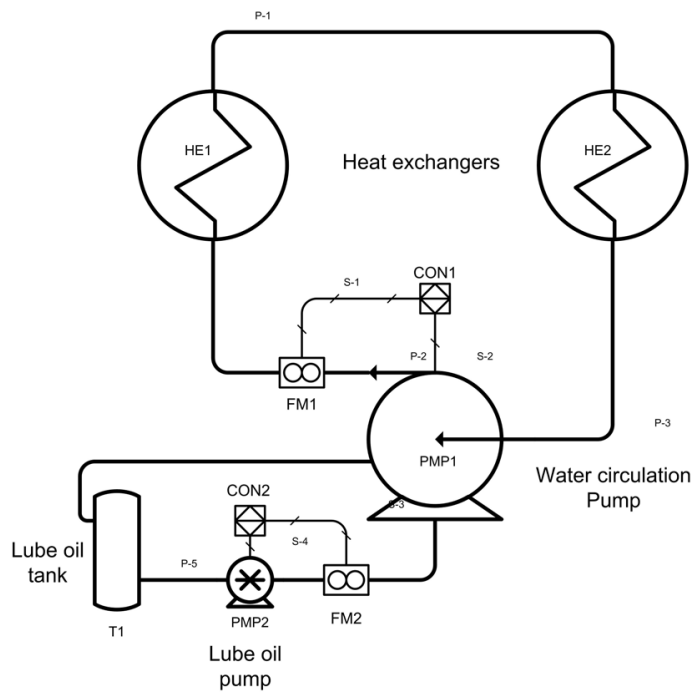

Fig. 2. The MFM model example

a) The MFM model: The model in Figure 3 represents the objectives and functions of a water circulation loop in 
a heat transfer system as they are represented in MFM. The example illustrates how the MFM model provides a comprehensive understanding of the purpose and functions of the circulation loop and its subsystems. On an overall level the model can be seen as composed of three sub-models representing different views on the water circulation system.

The first view (starting from the top) represents systems aspects related to water circulation and comprises the flow structure labeled MFS1, a maintain relation and the objective O1. This part of the models represents the overall objective of the water circulation, which is to maintain a flow of water. The flow structure contains the functions provided to circulate the water. In this simplified model the transport function T1 is the means used for water circulation.

The second view is partially overlapping with the first view because what is seen here as a means (the transport T1) is in the second view seen as an end. Transport $\mathrm{T} 1$ is related to the means of transport which is the pumping represented by the energy flow structure EFS1. T1 and EFS1 are related by a type of means-end relation called a producer-product relation in MFM. The flow structure EFS1 is decomposed into the flow functions representing the services provided by components of the pump system (including the energy supply) in order to achieve the end, the transportation of water represented by $\mathrm{T} 1$.

The third view is related with the second view through the energy transport $\mathrm{T} 2$, an enable relation and an associated objective $\mathrm{O} 2$ which is the end to be maintained by the functions contained in the flow structure MFS2. The flow structure MFS2 represents the functions involved in the lubrication of the pump and the objective $\mathrm{O} 2$ represents the condition that should be fulfilled in order to ensure that the pump is properly lubricated. A condition which should be satisfied in order to enable the pump to provide its functions. The flow functions inside MFS2 accordingly represent the functions of the pump lubrication system.

Even though the simple example does not utilize all the concepts of MFM, it demonstrates the power of MFM to represent in a clear and logical way relations between the goals and functions of a system. The MFM modeling language has a strong syntax which defines rules for combining the different entities and relations of the language into a consistent model.

The model in Figure 3 show the functions of the components and subsystem which contributed to the overall objective of the system (deliver water flow). No consideration was accordingly given to the purpose and function of control systems in meeting this objective. As is well known control systems are important for ensuring that process objectives are met in spite of uncertainty and disturbances in the process. MFM has a set of functions which can be used to represent control system functions (see Figure 1).

\section{INTELLIGENT CONTROL OF POWER SYSTEMS}

The overall purpose of intelligent control of power system is to detect and interpret the significance of deviations in power system states from their normal expected values and to provide an appropriate remedial action to restore normal

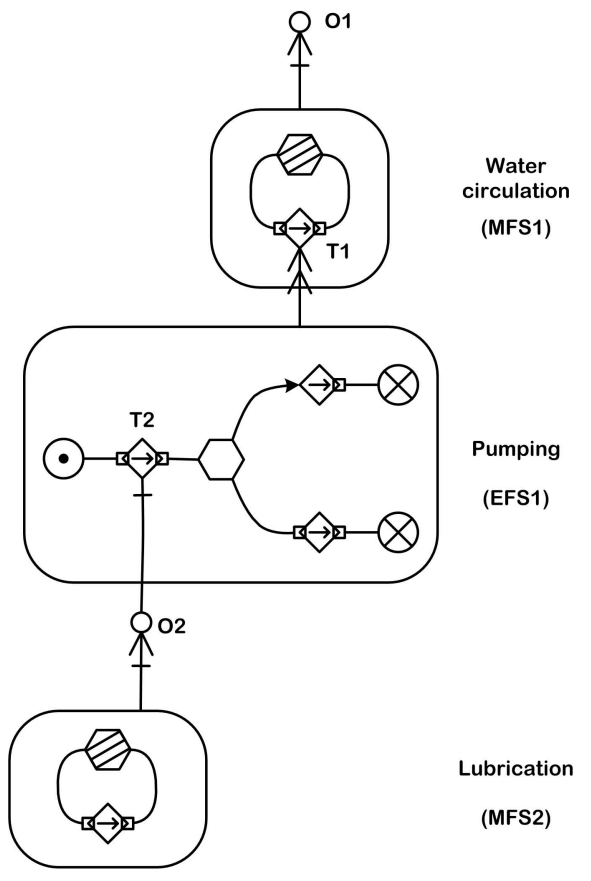

Fig. 3. The MFM model example

or safe operation. Usually, several interpretations are possible of a given situation depending on the specific goal that may be dependent on the situation. Three main types of operating goals can be distinguished.

(1) In some situations the goal is to relate the symptoms of a power system disturbance to a possible failed component or subsystem. In this case the goal of the intelligent controller is to exchange the failed component or utilize redundancy. It is clear that such a goal would only be acceptable, if the controller is allowed to take the failed entity out of service and that there is enough time to make the repair.

(2) These conditions are not met if there are no spare parts or alternatives or if overall requirements to power system operation cannot be satisfied during the period of change. In such situations it would be necessary to find means of compensation for the disturbance that avoid the removal of the failed component.

(3) However, in situations with high risk and uncertainty it can even be a dangerous decision to compensate the disturbance. Under such circumstances the goal of the intelligent control should be to derive and evaluate possible consequences of the disturbance and to provide protective action (e.g. shut down). In this case the decision to act could be done without knowing the prime cause of the failure. Taking into account the uncertainty and the possible risks this may be the best strategy.

These examples illustrate a variety of problems that typically should be handled by an intelligent power control system. In order to satisfy these demands the control system must maintain an overview of the situation in order to choose a proper control strategy and decide how to act. Skilled human 
supervisors that can keep the power system running under a variety of disturbance situations, have apparently such an ability to adapt their control actions to the actual power system operating situation. This capability is difficult to model and simulate in artificial intelligence programs because of the range of situations to be considered and because of the difficulties of defining the strategies that control the interdependent concurrent reasoning processes that are required.

The modeling problem is further complicated by the fact that power system knowledge required for the intelligent control would be dependent on the overall operational goal. If the goal is to compensate for a failed component there would be a need for knowing possible redundant standby components or other means to remediate the failure. If the goal is to protect the power system operation in order to ensure reliability of supply, knowledge about means of protection would be required. The power system knowledge to be used is therefore dependent on the task to be solved, i.e. it is determined by an interpretation of the power system physical features within a task context.

\section{A. The Power System Example}

For the purpose of a study case, we take an example from a distribution network. Figure 4 shows a single line diagram of the example. It consists of three local DGs, four (aggregated) loads and a connection to the utility grid. It is assumed that both loads and DGs are controlled by smart controllers (Load and DG agents), and that there is a regulator agent, responsible for overall balancing. These agents have the capability to react to changes in environment and choose appropriate action to respond the the changes. We consider the scenario when this part of the network is disconnected form the main utility grid. The role of the regulator agent is to maintain overall power balance in this isolated part of network. The DG agents may provide regulation service by delivering extra active power. The load agents continuously monitor voltage at their nodes, and if find any disturbance, they start looking for availability of regulation services.

1) A control situation: In the following we consider a control situation in the power system as depicted in Table I and show that a representation of the power system in an MFM model can be used to represent the perspectives of the three agents and to reason or negotiate about alternative control actions for the same situation. Table I depict the goals of the regulator, load and DG agents and show that each agent has a different interpretation of the same situation depending on the goal. The table show also that there are four different ways of responding in order to control the situation.

\section{B. MFM model of the Power System Example}

Figure 5 present a MFM model of the power system example based on the modeling principles presented above. The model contains three views of the power system: an overall systems's view, the view of one of the DG agents (DG3 agent) and a load agent (L4 agent). Views for the other generators and loads are not included for simplicity of the presentation. In the

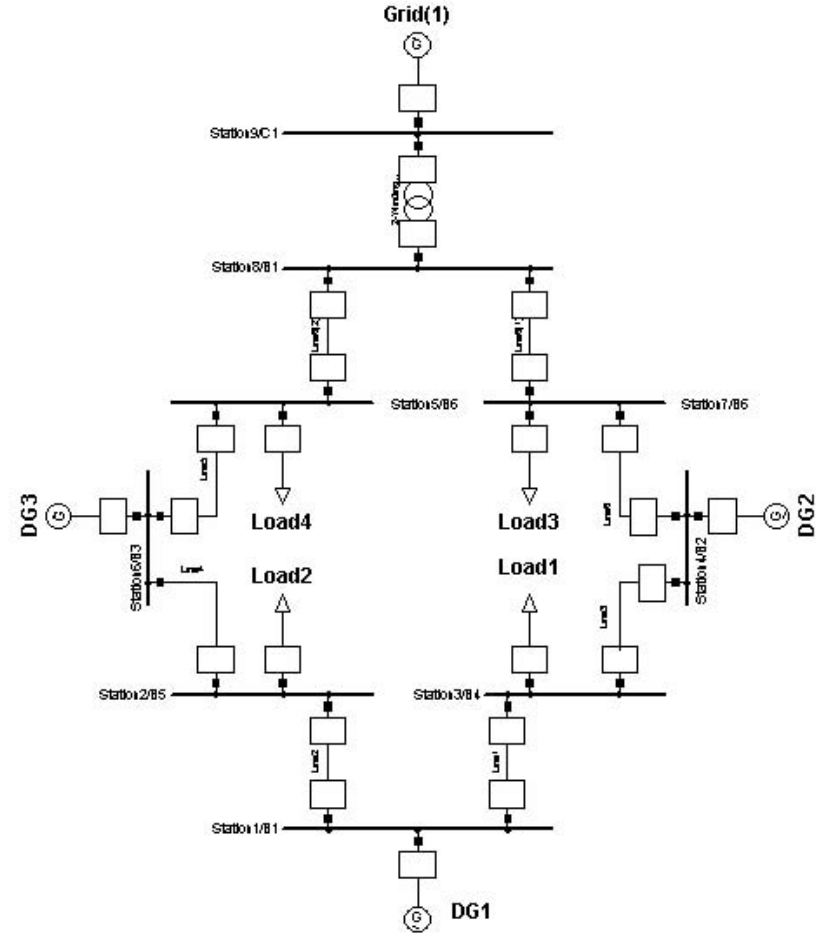

Fig. 4. The power system example

following we will describe the model and demonstrate that the model provides a coherent representation of the different views of the agents and a representation of the relations between the views which can be used for reasoning about alternative control actions. The example and the MFM model has also been discussed in [5], [22]. A detailed discussion of views and perspectives in MFM with power system examples is presented in [4].

1) System's view - overall balancing: The part of the model comprising G1, $F_{S C H 1}, \mathrm{G} 2$ and $F_{S C H} 2$ represents the view of system - related to the task of overall balancing. This is a view of regulation of grid resources. Grid resources comprises three distributed generators represented by MFM source functions $S o_{D G 1}, S o_{D G 2}$ and $S o_{D G 3}$ and four loads represented by MFM sink functions $S i_{L 1}, S i_{L 2}, S i_{L 3}$ and $S i_{L 4}$. Furthermore the storage function labeled $S t$ represents the total rotating inertia in the system. The functions included in the flow structure represent accordingly the resources involved in the balancing of power in the example case. The transfer of power from the generators to the loads is represented in MFM by the transport functions $T r_{D G 1}, T r_{D G 2}, T r_{D G 3}$, $T r_{L 1}, T r_{L 2}, \operatorname{Tr}_{L 3}, \operatorname{Tr}_{L 4}$. Since the control strategy adopted is decentralized, this view gets realized by the individual actions of agents.

2) The view of DGs: The view of DG3 is representing how the generator agent sees the control situation. From the perspective of the system, DG3 is simply a power source $S_{o_{D G 3}}$. But from the perspective of the generator agent, the grid is a power consumer or sink represented by $S i_{D G 3}$ and the 
TABLE I

THE INTERPRETATION OF A POWER SYSTEM STATE AND THE CONTROL ACTION TO BE TAKEN DEPENDS ON THE AGENTS GOAL. THE SAME SITUATION OF IMBALANCE MAY THEREFORE CALL FOR DIFFERENT ACTIONŤS DEPENDING OF THE AGENT

\begin{tabular}{l|l|l|l}
\hline Agent & Goal & State & Control intention \\
\hline Regulator & overall balancing & load-demand imbalance & dispatch new set-points to DGs \\
\hline L4 Agent (global perspective) & voltage stability at node & voltage drooped at node & look for regulation service \\
\hline L4 Agent (local perspective) & consumption of required power & $\begin{array}{l}\text { un-availability of required } \\
\text { power }\end{array}$ & request more active power \\
\hline $\begin{array}{l}\text { DG3 Agent (global perspec- } \\
\text { tive) }\end{array}$ & deliver of power to network & frequency drop at node & inertia response \\
\hline DG3 Agent(local perspective) & $\begin{array}{l}\text { maximize production / earn } \\
\text { profit }\end{array}$ & $\begin{array}{l}\text { demand for more power from provide more power } \\
\text { network }\end{array}$ \\
\hline
\end{tabular}

power source feeding the generator is $\mathrm{So}_{D G 3}$. The inertia of generator DG3 is represented by an energy storage function $S t_{D G 3}$. The goal to be achieved by the generator agent is represented by $G_{D G 3}$. The goal specifies the power to be delivered to the grid.

3) The view of L4: The view of L4 is representing how the load agent may see the control situation. From the perspective of the system, L4 is simply a power consumer or load $S i_{L 4}$. But from the perspective of the load agent, the grid is a power source represented by $S_{L 4}$ and the power consumer is represented by $S i_{L 4}^{1}$. Note that $S i_{L 4}$ in $F_{S C H 1}$ is not the same as $S i_{L 4}^{1}$ in $F_{L 4}$. The conversion of the power in the load from the electric energy e.g. to another form of energy is represented by the conversion function $C n_{L 4}$.

4) Relations between the three views: The relations between the views are indicated above. However, the MFM language allows systematic expansion and aggregation of functions so that e.g. the system's view may be expanded by incorporating the views of DG3 and/or L4. In a service oriented agent architecture, this expansion could be done either as a demand from the system or could be done by the DG3 and L4 agents explaining how they see the situation.

\section{Representing the control situations in MFM}

The imbalance situation and its interpretations by the three agents presented above in table can be expressed explicitly by the MFM model in Figure 5. How this is done will be explained briefly in the following.

1) The regulator agent: The goal of the regulator agent is to ensure overall balancing. With his goal in view the agent will perceive the situation as a load-demand imbalance. The imbalance can be expressed in the flow structure $F_{S C H 1}$ as a deviation from the normal pattern of energy flows delivered by the three sources $S o_{D G 1}, S o_{D G 2}, S o_{D G 3}$ and consumed by the four sinks $S i_{L 1}, S i_{L 2}, S i_{L 3}, S i_{L 4}$. Within the view of the regulator agent the control action will be to restore the situation by dispatching new set points $G_{D G 1}, G_{D G 2}, G_{D G 3}$ to the DG's.

2) The agent L4: The agent Load4 has two alternative goals as shown in Table I. Depending on the goal chosen the agent will take appropriate action.

If the goal is to ensure voltage stability and the situation therefore is interpreted as a voltage droop problem, the control action of the agent is to request a regulation service. The

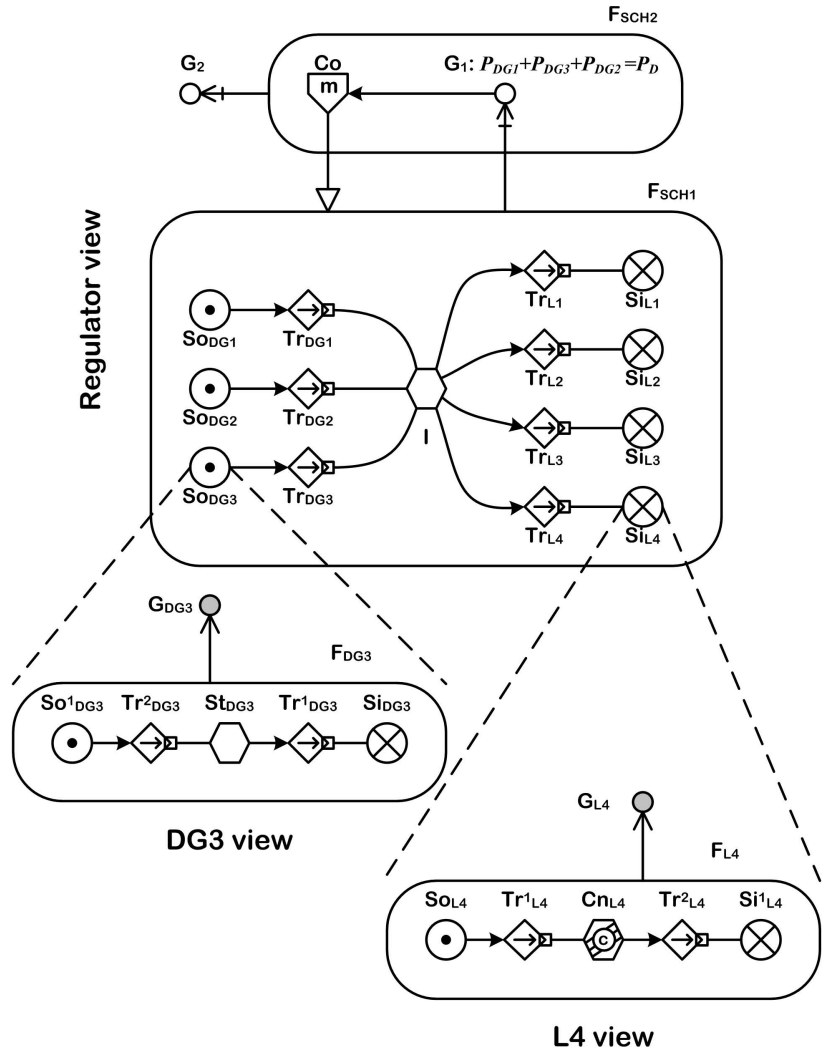

Fig. 5. The views of the regulator, generator and load agents represented in MFM

voltage is here seen as an attribute of the source $S_{L} o_{4}$ which represent the network as seen in the view of L4.

If the goal of the agent is to ensure consumption of required power and the situation therefore in this case is interpreted as a problem if unavailability of required power, the control action of the agent is to request more active power. The power is here seen as an attribute of the source.

3) The agent DG3: The agent DG3 has also two alternative goals as shown in Table I and again depending on the goal chosen the agent will take appropriate action.

If the goal is to deliver power to the network and the situation therefore is interpreted as a frequency drop problem, the control action of the agent is to execute an inertia response. The inertia is represented by the storage function $S t_{D G 3}$ and the response will be a temporary increase in the power flow 
represented by the transport $\operatorname{Tr}_{D G 3}$ caused by an increase in the energy stored by $S t_{D G 3}$. This causal relation is represented in the MFM model by an arrow pointing towards the transport function (the agent relation shown in Figure 1).

If the goal of the DG3 agent is to maximize production in order to earn profit and the situation therefore is seen as a demand for more power from the network, the control response will be to provide more power by increasing the flow attributed to the source $S o_{D G 3}$. The network is in this view seen as a sink $S i_{D G 3}$ and the power demanded is an attribute of this function.

\section{CONCLUSIONS}

Work presented in this paper presents the problem of interpretation in complex control situations of electric power systems. The importance of being able to reason explicitly about different views on a control situation is explained. It is shown that Multilevel Flow Modeling can provide model based support to explicit means-ends reasoning and handling of views. The application of explicit means-ends models provides a novel extension of the classic belief-desire-intention BDI paradigm of multiagent systems. A power system example demonstrate the importance of means-end and part-whole concepts in modeling and intelligent control of complex power systems.

\section{ACKNOWLEDGEMENT}

This work was supported partly by the Danish PSO Energy Research Program.

\section{REFERENCES}

[1] K. V. Gernaey, M. Lind, and S. B. Jørgensen. Towards understanding the role and function of regulatory networks in microorganisms. In L. Puigjaner and G. Heyen, editors, Computer Aided Process \& Product Engineering. Wiley-VCH, Weinheim, Germany, 2004.

[2] A. Gofuku and Y. Tanaka. Development of an Operator Advisory System: Finding Possible Counter Actions in Anomalous Situations. In Proc. 5'th International Workshop on Functional Modeling of Complex Technical Systems, pages 87-97, Paris, France, July 1-3 1997.

[3] A. Gofuku and Y. Tanaka. Application of Derivation Technique of Possible Counter Actions to an Oil Refinery Plant. In Proc. 4'th IJCAI Workshop on Engineering Problems for Qualitative Reasoning, pages 77-83, Stockholm, 1999.

[4] K. Heussen and M. Lind. Integration of power systems with other energy systems using multilevel flow modeling of control. In Proceedings International Summer School Symposium on Energy Science for Young Generations (ISSES-YGN), Kyoto Japan, August 20-22 2009. Kyoto University Global Center of Excellence of Energy Science.

[5] K. Heussen, A. Saleem, and M. Lind. Control architecture of power systems: Modeling of purpose and function. In Proceedings IEEE PES General Meeting, Calgary, Alberta Canada, 26-30 July, 20092009.

[6] M. N. Larsen. Deriving Action Sequences for Start-Up Using Multilevel Flow Models. PhD thesis, Department of Automation, Technical University of Denmark, 1993.

[7] J. E. Larsson. Diagnosis based on explicit means-end models. Artificial Intelligence, 80(1):29-93, 1996.

[8] M. Lind. The use of flow models for design of plant operating procedures. In Proc. IWG/NPPCI Specialist meeting on procedures and systems for assisting an operator in normal and anomalous nuclear power plant operation situations, Garching, Federal Republic of Germany, December 1979.

[9] M. Lind. The use of flow models for automated plant diagnosis. In J. Rasmussen and W. B. Rouse, editors, Human Detection and Diagnosis of System Failures. Plenum Press, New York, 1981.
[10] M. Lind. Modeling goals and functions of complex industrial plant. Applied Artificial Intelligence, 8(2):259-283, 1994.

[11] M. Lind. Status and challenges of intelligent plant control. In Proc. 4'th IFAC Symposium on Dynamics and Control of Chemical Reactors, destillation Columns and Batch Processes (DYCORD+95), Helsingør, Denmark, June 7-9 1995.

[12] M. Lind. Interpretation problems in modelling complex artifacts for diagnosis. In Proc. Cognitive Engineering for Process Control (CSEPC'96), Kyoto, Japan, November 12-15 1996.

[13] M. Lind. Plant modeling for human supervisory control. Transactions of the Institute of Measurement and Control, 21(4-5):171-180, 1999.

[14] M. Lind. Modeling goals and functions of control and safety systems theoretical foundations and extensions of MFM. NKS-R-07 project report, Ørsted DTU, Technical University of Denmark, DK 2800 Kongens Lyngby, Denmark, September 112005.

[15] M. Lind. The what, why and how of functional modelling. In Proceedings of International Symposium on Symbiotic Nuclear Power Systems for the 21'st Century (ISSNP), pages 174-179, Tsuruga, Japan, July 9-11 2007.

[16] M. Lind. Perspectives on multilevel flow modeling. In Proc. 4.th International Symposium on Cognitive System Engineering Approach to Power Plant Control (CSEPC2008), Harbin , Heilongiiang China, September 8-10 2008

[17] J. Liu, H. Yoshikawa, and Y. Zhou. Application of multilevel flow modeling to describe complex processes in a nuclear fuel cycle. In Proceedings CSEPC 2004 Cognitive Systems Engineering in Process Control, pages 114-120, Sendai, Japan, November 4-5 2004.

[18] J. Ouyang, M. Yang, H. Yoshikawa, Y. Zhou, and J. Liu. Alarm Analysis and Supervisory Control Plan of PWR Plant. In Proceedings of CSEPC 2004, Cognitive Systems Engineering in Process Control, pages 61-68, Sendai, Japan, Nocember 4-5 2004.

[19] J. Petersen. Situation assessment of complex dynamic systems using MFM. In Proceedings of 8th. IFAC/IFIP/IFPRS/IEA Symposium on Analysis, Design and Evaluation of Human-Machine Systems, pages 645-650, Kassel, Germany, September 18-20 2001.

[20] L. Wass Petersen. Multilevel flow model of heat integrated distillation plant. MSc thesis, Ørsted DTU, Automation, 2005.

[21] N. L. Rossing, M.Lind, N. Jensen, and S. B. Jørgensen. A goal based methodology for hazop analysis. In Proc. 4.th International Symposium on Cognitive System Engineering Approach to Power Plant Control (CSEPC2008), Harbin, Heilongjiang, China, September 8-10 2008.

[22] A. Saleem, K. Heussen, and M. Lind. Agent services for situation aware control of power systems with distributed generation. In Proceedings IEEE PES General Meeting, Calgary, Alberta Canada, 26-30 July, 2009 2009.

[23] A. Saleem, T. Us, and M. Lind. Means-end based functional modeling for intelligent control: Modeling and experiments with an industrial heat pump. In Proc. IASTED conference on Intelligent Control Systems (ICS2007), Cambridge, Massachussets, USA, Nivember 21-23 2007.

[24] T. Us, N. Jensen, M. Lind, and S. B. Jørgensen. Fundamental principles of alarm design. In Proc. 4.th International Symposium on Cognitive System Engineering Approach to Power Plant Control (CSEPC2008), Harbin, Heilongjiang China, September 8-10 2008. 